\title{
The fire vulnerability evaluation of the old building based on fuzzy
}

\section{comprehensive assessment method}

\author{
Xiaodong Chen ${ }^{1, a^{*}}$, Yongjun Liu ${ }^{2, b}$, Yangyang $\mathrm{Liu}^{3, \mathrm{c}}$ \\ ${ }^{1}$ Shenyang Jianzhu University, Shenyang City, Liaoning Province, China \\ ${ }^{2}$ Shenyang Jianzhu University, Shenyang City, Liaoning Province, China \\ ${ }^{3}$ Shenyang Jianzhu University, Shenyang City, Liaoning Province, China

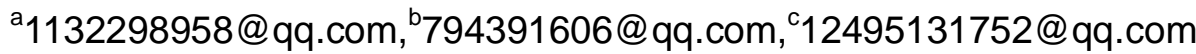

Keywords:fire hazard; old building; vulnerability; fuzzy comprehensive assessment Abstract:In order to assess fire vulnerability of old buildings quickly and reasonably, a model for an assessment system of fire vulnerability of old buildings was built, based on the theory of fuzzy comprehensive assessment. Based on the analysis of the building fire spreading process and the mechanical properties of building materials under high temperature index factors of the old buildings fire vulnerability assessment model were determined at different levels, and analytic hierarchy process (AHP) was used to determine the weight coefficients of the model at the same level factors. A software for fire vulnerability assessment of old buildings was developed with the help of Visual Basic 6.0 on Windows platform to assess fire vulnerability of old buildings. Using the developed software, the fire vulnerability assessment for an old hotel was carried out. It is shown that assessment results for the fire developments of one, two and three are not vulnerable but four and five are serious vulnerable.

Fire hazard is burning without control in time and space. It is one of the main disasters in the world and also causes loss of life and property as one of the most direct and serious disasters[1]. According to the statistics ,since 2000, fire hazard in China occurred two hundred and two thousand ,2557 dead and 3671 injured on the average annual, when the losses were up to 1.5 billion yuan directly[2]. Especially building fire, it accounts for about $70 \%$ of total annual fire. The fire of building has become an important threat for casualties and property losses.

The total construction area in our country is at the top of the world. According to statistics, existing buildings in our country is more than 45 billion square meters[3]. In view of the old buildings have been used for long time, the structure bearing capacity began to degenerate, fire control facilities appear to be different damaged condition, so it has become a severely afflicted area of building fire. However, the domestic research on old buildings fire vulnerability assessment is few. Therefore, the author through the study of the influence of old reinforced concrete frame structure vulnerability factors, the old building fire vulnerability assessment model was built based on the theory of fuzzy comprehensive assessment to assess the old building fire vulnerability quickly and effectively to provide reliable information for the old buildings fire vulnerability and structures detection ,reinforcement and reconstruction.

\section{Vulnerability assessment model}

\section{The basic theory of fuzzy comprehensive evaluation method}

Comprehensive evaluation is defined according to the intended purpose, and determine the research object attributes (metrics), and the attributes is transformed into the design objective and 
quantitative values or subjective utility behavior[4]. It need to be considered a series of factors, and is not easy to accurately judge them on effects of things in the evaluation results, especially when there are more factors, so application of fuzzy mathematics method is suitable.

The main steps of fuzzy comprehensive assessment are as follows:

(1) single factor evaluation

Fuzzy mapping is given

$$
\begin{aligned}
& f: U \rightarrow f(\mathrm{~V}) . \\
& u_{i} \rightarrow f\left(u_{i}\right)=\left(r_{i_{1}}, r_{i_{2}}, \mathrm{~L}, r_{i_{n}}\right) .
\end{aligned}
$$

$U$ is the factors set, it regards the factors of assessment objects as a set of common elements. As $V$ is the evaluation set that the results of assessment can be divided into certain levels(depending on the specific issues and regulations to differentiate).

(2) comprehensive evaluation transformation matrix

$$
R=R_{f}=\left(r_{i j}\right)_{m \times n} .
$$

(3) comprehensive assessment

$$
B=A \circ R=\left(b_{1}, b_{2}, \mathrm{~L}, b_{n}\right) .
$$

\section{Vulnerability assessment method}

\section{Evaluation grading}

Under the function of building structure fire vulnerability refers to the building structure in different fire intensities occur some damage possibility. Building fire vulnerability degree, according to disruption effects, will be divided into four grades: no vulnerability, mild vulnerability, moderate vulnerability, severe vulnerability.

The old building vulnerability evaluation index

Through research and analysis of architectural features and behavior characteristics of old buildings, influencing factors of building vulnerability can be divided into two categories. Fire intensity U1: fire load density, fire location, fire type, fire area ventilation, fire development situation; Structure fire resistance U2: building age, building fireproof rank, building component information, reinforcement, building suffered disasters.

\section{To determine the single factor membership function}

For non quantitative factors. in this paper, based on the analogy method to establish the membership function, for the observed level of a factor of four grade (not vulnerable, mild damage, moderate vulnerability, severe damage, can choose the language with five to meet the following degree: in full compliance with, in line with the basic, some in line, not too conforms to, has nothing to do with, while give the corresponding numerical: $1,0.75,0.5,0.25,0$.Through the above method, you can determine the membership functions of the non quantitative factors. To determine the membership functions of the fire type as an example, according to the classification of the NFPA, the fire development rate is divided into very fast, fast, medium and slow, you can get membership of fire types, as shown in Table 1 shows. Similarly, the membership function can be determined for the other single factor indictors. 
Table 1 The membership function of fire style

\begin{tabular}{ccccc}
\hline fire type & no & mild & moderate & Severe \\
\hline very fast & 0 & 0 & 0 & 1 \\
fast & 0 & 0 & 0.25 & 0.75 \\
medium & 0 & 0.25 & 0.75 & 0 \\
slow & 0.25 & 0.75 & 0 & 0 \\
\hline
\end{tabular}

Fuzzy comprehensive assessment method

(1)primary comprehensive assessment

Fire type of evaluation matrix, for example, the establishment of the single factor subordinate function, obtain the factors of I, II, III, IV after four grades of membership degree, then structure factors evaluation matrix.

$$
R_{13}=\left[\begin{array}{l}
\mu_{1} \\
\mu_{2} \\
\mu_{3} \\
\mu_{4}
\end{array}\right] \circ\left[\begin{array}{llll}
\mu_{1 \mathrm{I}} & \mu_{1 \mathrm{II}} & \mu_{1 \mathrm{III}} & \mu_{1 \mathrm{IV}} \\
\mu_{2 \mathrm{I}} & \mu_{2 \mathrm{II}} & \mu_{2 \mathrm{III}} & \mu_{2 \mathrm{IV}} \\
\mu_{3 \mathrm{I}} & \mu_{3 \mathrm{II}} & \mu_{3 \mathrm{III}} & \mu_{3 \mathrm{IV}} \\
\mu_{4 \mathrm{I}} & \mu_{4 \mathrm{II}} & \mu_{4 \mathrm{III}} & \mu_{4 \mathrm{IV}}
\end{array}\right]=\left[r_{i j}\right]_{4 \times 4} .
$$

(2) The secondary comprehensive assessment

(1) fire intensity information

Depending on the type of fire load density, fire location, fire type, fire area ventilation, fire development situation in five aspects of membership function, we can obtain each factors on I, II, III, IV of four grades of membership degree, then build factors evaluation matrix:

$$
R=\left[\begin{array}{l}
\mu_{1} \\
\mu_{2} \\
\mu_{3} \\
\mu_{4} \\
\mu_{5}
\end{array}\right]=\left[\begin{array}{llll}
\mu_{1 \mathrm{I}} & \mu_{1 \mathrm{II}} & \mu_{1 \mathrm{III}} & \mu_{1 \mathrm{IV}} \\
\mu_{2 \mathrm{I}} & \mu_{2 \mathrm{II}} & \mu_{2 \mathrm{III}} & \mu_{2 \mathrm{~V}} \\
\mu_{31} & \mu_{3 \mathrm{II}} & \mu_{3 \mathrm{III}} & \mu_{3 \mathrm{IV}} \\
\mu_{4 \mathrm{I}} & \mu_{4 \mathrm{II}} & \mu_{4 \mathrm{III}} & \mu_{4 \mathrm{~V}} \\
\mu_{5 \mathrm{I}} & \mu_{5 \mathrm{II}} & \mu_{5 \mathrm{III}} & \mu_{5 \mathrm{~N}}
\end{array}\right]=\left[r_{i j}\right]_{5 \times 4} .
$$

(2) structure fire resistance information

Primary comprehensive evaluation conclusion $B_{21}, B_{22}, B_{23}, B_{24}, B_{25}$ of building age, buildi ng fireproof rank, the building component fire resistance ability, building whether reinforcem ent, building suffered disasters constitute basic information of building evaluation matrix $R_{2}$,

$R_{2}$ then through the formula (7), get the basic information about building the evaluation co nclusion $\mathrm{B}_{2}$.

$$
B_{2}=A_{2} \mathrm{O} R_{2} \text {. }
$$

(3)the third comprehensive assessment

According to the second level of each subsystem evaluation conclusion B1、B2 structure composed of old reinforced concrete frame structure vulnerability overall evaluation matrix $\mathrm{R}$.

$$
R=\left[\begin{array}{l}
B_{1} \\
B_{2}
\end{array}\right]=\left[\begin{array}{l}
A_{1} \mathrm{o} R_{1} \\
A_{2} \mathrm{o} R_{2}
\end{array}\right]=\left[\mathrm{r}_{i j}\right]_{2 \times 4} .
$$

\section{Index weight determining}

Using analytic hierarchy process (AHP) to calculate the weight of evaluation factors, can make the complex problems hierarchically, simple, greatly and reduce the influence of subjective factors, and the author here do not introduce. Its advantage is the combination of quantitative and qualitative, has high logicality, systematization, conciseness and practicability, is for large system, an effective method of multi-level and multi-objective decision problem[5]. At the same time the introduction of scaling the 1-9 ratio[6], we can build the consistency of structural indicators at all levels of 
the evaluation matrix and check evaluation matrix.

\section{The evaluation results processing}

In this paper, it can use the method of fuzzy distribution method and the maximum membership degree with the results on the final evaluation index $B$.

\section{Software development}

Based on the application system Windows, with the aid of programming software Visual Basic 6.0, according to the established evaluation model, using the fuzzy comprehensive assessment method, the "old reinforced concrete frame structures under fire vulnerability assessment system" is developed.

\section{Software applications}

\section{Building basic situation}

YanDou hotel in Chaoyang city, for example, the building type ventilation is in good condition, building age is for 10 years, the protective layer thickness of the beam is $30 \mathrm{~mm}$, floor covering thickness is $10 \mathrm{~mm}$, stele of protective layer thickness is $30 \mathrm{~mm}$, the protective layer thickness of the side column is to $40 \mathrm{~mm}$, and aggregate types of siliceous concrete aggregate, skirt room some columns and beams reinforced, fireproof rank for level 1.

Fire scenario Settings

Evaluation of a building fire vulnerability should be discussed under different fire scenario. In this paper, select a typical fire scenario here for discussion. It is considered that the first floor hall in the middle of the building fire: due to the structure of the hall, the layer is steel structure when the fire broke out the possibility of larger to make vulnerable, so the center of the fire on the first floor hall fire is a kind of fire scenario.

\section{Fire development}

Fire spread with uncertainty, if in the fire just starting to happen in time to fight, so for the construction of the impact is very small; if the fire is not under control, crashing, then fire will increasingly large and difficult to control. Software gives five different fire development fire scenarios. In the old building fire vulnerability of evaluation you can discuss the spread in the five different fire development under the situation of building fire vulnerability,.

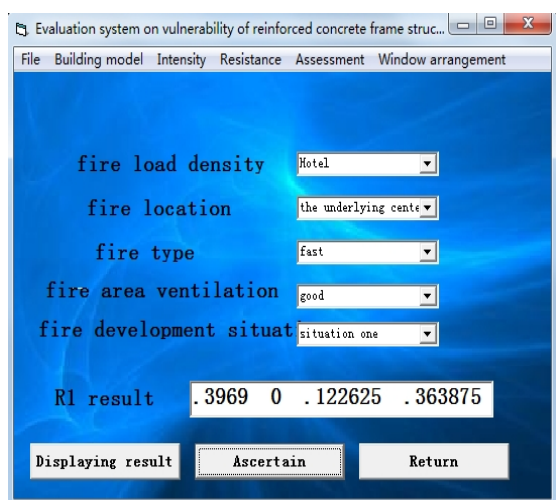

Fig. 1 The evaluation result in scene one

\section{Fire vulnerability assessment}

(1)For fire intensity information entry, firstly consider the development situation of fire intensity in five kinds of fire to get fire intensity information of judgment matrix, here only the evaluation results of fire development situation one is given, as shown in figure 1. 
(2)The hotel's structure fire resistance information is respectively recorded in the corresponding information the module, as shown in figure 2, to calculate the fire resistance ability of the construction of evaluation matrix. The results is shown in figure 3.
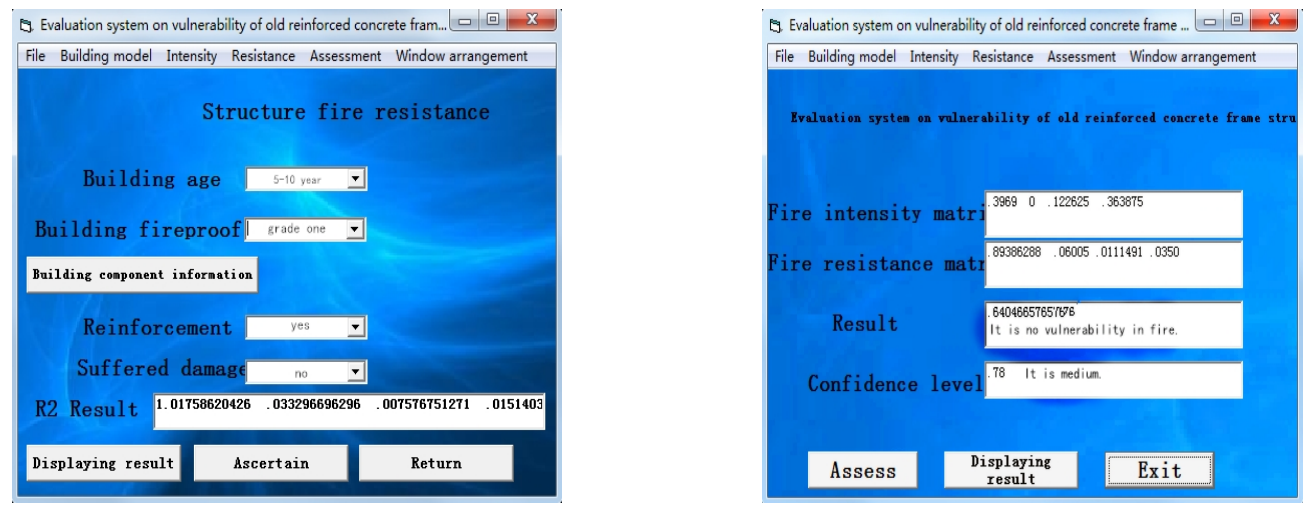

Fig. 2 The interface of the structure fire resistance Fig. 3 The evaluation result in scene one

\section{The evaluation results of analysis}

Through the evaluation result in five different fire development situations, there will be significantly different fire intensity. In the first, second, third fire development situation, fire intensity is not very large, and the structure fire resistance is very strong, so the evaluation result is not vulnerable; in the fourth, fifth fire development situation, the fire began to spread and fire intensity got larger, in the same kind of structure under the condition of fire resistance. It is easy to damage, so the evaluation result is serious vulnerability.

\section{Summary}

(1)By running the software to evaluate the vulnerability of an old hotel, you can see that the written software can reasonably reflect the hotel fire vulnerability of the status quo based on the hotel fire vulnerability assessment model. This shows that the setting of the fire scene and the analysis of old hotel fire five development finally to use the old hotel overall fire vulnerability of assessment method is feasible.

(2)Using VB6.0 by use of language to write the fire vulnerability assessment software operability is strong and get reliable results, successfully solving the shortcoming of time-consuming long and prone to mistake, achieved the purpose of rapid evaluation of of the old hotel fire vulnerability.

\section{Reference}

[1]LI Haijiang. 2000-2008 national super-large fire statistical analysis [J]. Journal of fire science, 2010, 18(1).

[2]Ma Yongzhen, Wu Lurong. Chinese fire optimal grey regression combination forecasting model [J]. China Safety Science Journal, 2006, January, Volume 16 first.

[3]Zhu Chunyi. The economic analysis of building structure reinforcement technology [D].Nanchan: East China Jiaotong University,2012.

[4]Jiang Gao. Fuzzy hierarchy comprehensive evaluation method and its application [D]. Tianjin: Tianjin university, 2005.

[5]Salty T L. Modeling unstructured decision problem the theory of analytical hierarchies [J].Math Compute Simulation, 1978 (20): 147-158. 
[6]Liu Yongjun, Zhang Zhenwen, Chen Xiaodong, Liu Yangyang. Based on the fuzzy gray theory of an old hotel fire vulnerability evaluation [J]. Journal of Shenyang Jianzhu University, 2014, 30(5). 